Sektion Forschung und Lehre in der Pflege, Institut für Sozialmedizin und Epidemiologie, Universität zu Lübeck Sascha Köpke (Prof. Dr. phil.), Frauke Koch, Anja Behncke (MSc), Katrin Balzer (Dipl.-Pflegepäd.)

\title{
Einstellungen Pflegender in deutschen Kranken- häusern zu einer evidenzbasierten Pflegepraxis
}

- Was ist (zu dieser Thematik) schon bekannt?

Einstellungen von Pflegenden sind von besonderer Bedeutung bei

der Implementierung einer evidenzbasierten Pflegepraxis.

Was ist neu?

Pflegende bestätigen den Wert von Forschung für die eigene Praxis, es fehlt jedoch an Grundvoraussetzungen für eine evidenzbasierte Pflegepraxis, wie der Nutzung aktueller wissenschafticher Fachinformationen, um relevante Forschung zu identifizieren und zu implementieren.

- Welche Konsequenzen haben die Ergebnisse für die Pflegepraxis? Die Aus- und Weiterbildung Pflegender sollte im Sinne einer evidenzbasierten Pflegepraxis angepasst werden.

Die Bedeutung der Einstellung Pflegender für die Etablierung einer evidenzbasierten Pflegepraxis (EBP) ist international gut belegt. Für den deutschsprachigen Raum liegen bislang nur wenige Daten vor. Diese Querschnittstudie zielt darauf, pflegerische Sichtweisen auf relevante Kontextfaktoren für die Implementierung einer EBP zu erfassen. Hierfür wurden mithilfe eines selbst entwickelten Fragebogens auf Basis etablierter Instrumente in 21 norddeutschen Krankenhäusern 1384 Pflegende befragt, von denen 1023 (74\%) antworteten. Die Ergebnisse zeigen eine prinzipiell positive Einstellung gegenüber EBP. Die Mehrheit der Befragten erachtet Forschung als relevant für die Praxis. Unterstützung durch Vorgesetzte sowie Kolleg(inn)en wird als wichtige Voraussetzung gesehen. Die Umsetzung gelingt jedoch nur unzureichend. So sind Pflegende nicht über aktuelle Forschungsergebnisse informiert. Primärliteratur wird kaum genutzt.

Manuskript eingereicht am 19.9.2012 Manuskript akzeptiert am 5.2.2013
Lediglich eine Minderheit ist bereit, eigene Mittel für Tagungen aufzuwenden oder in naher Zukunft ein pflegewissenschaftliches Studium zu beginnen. Die Erhebung liefert erstmals für den deutschsprachigen Raum aussagekräftige Daten zur Einstellung Pflegender zu EBP. Pflegende bestätigen hierbei den Wert von Forschung für die eigene Praxis, es fehlt jedoch an Grundvoraussetzungen, beispielsweise der Nutzung aktueller wissenschaftlicher Fachinformationen, um relevante Forschung zu identifizieren und in die Praxis zu implementieren. Eine Ausrichtung der Ausbildung auf EBP, etwa im Rahmen von pflegewissenschaftlichen Studiengängen, erscheint in Deutschland überfällig.

Schlüsselwörter: evidenzbasierte Pflegepraxis, evidenzbasierte Pflege, Forschungsbasierung, Implementierung, Querschnittstudie

\section{Einleitung}

Für die Pflege, ebenso wie für die Medizin und andere Gesundheitsberufe, wird seit geraumer Zeit eine «evidenzbasierte Praxis (EBP)» gefordert, also eine Praxis, die auf validen wissenschaftlichen Erkenntnissen zur Wirksamkeit und Sicherheit pflegerischer und anderer Interventionen fußt (Meyer \& Köpke, 2012; Wallin, Bostrom \& Gustavsson, 2012). Seit mehr als einem Jahrzehnt wird im deutschsprachigem Raum das Konzept der evidenzbasierten Pflege (Evidence-based Nursing, EBN) diskutiert (Behrens \& Langer, 2004; Schlömer, 2000) und als grundlegend für eine moderne, wissenschaftlich fundierte Pflegepraxis angesehen (Meyer, Balzer \& Köpke, 2013). Diese Entwicklung bildet sich auch in veränderten Ausbildungscurricula in Deutschland und Österreich ab, die zumindest implizit eine EBP fordern (Breimaier, Halfens \& Lohrmann, 2011; Meyer \& Köpke 2012). Der Anspruch an eine EBP ist selbstverständlich, die klinische Versorgung zu verbessern, vor allem im Sinne einer positiven Beeinflussung patient(inn)enrelevanter Ergebnisse. Im Bereich der Pflege ist die Forschungslage hierzu nach wie vor nicht zufriedenstellend, jedoch deuten mehrere in den letzten Jahren publizierte Forschungsarbeiten darauf hin, dass durch EBP tatsächlich eine bessere Versorgung erreicht werden kann (Köpke, Mühlhauser, Gerlach, Haut, Haastert, Möhler et al., 2012; Middleton, McElduff, Ward, Grimshaw, Dale, D'Este et al., 2011; van Gaal, Schoonhoven, Mintjes, Borm, Koopmans \& van Achterberg, 2011). Dieses kann nur gelingen, wenn nachgewiesenermaßen wirksame Maßnahmen in die pflegerische Praxis implementiert werden. International wird hierfür häufig der Begriff «research utilization» verwandt. Dieses wird im Folgenden ebenfalls als EBP bezeichnet.

Eine Grundvoraussetzung dafür und somit für die Implementierung von EBP ist, neben der Entwicklung und Evaluation pflegerischer Interventio- 
nen, die Identifikation und Beseitigung von Barrieren der Umsetzung einer EBP. Hier gibt es bereits seit vielen Jahren umfangreiche Forschungsaktivitäten (Kajermo, Bostrom, Thompson, Hutchinson, Estabrooks \& Wallin, 2010; Hutchinson \& Johnston, 2004; Retsas, 2000; Sitzia, 2002). Die Ergebnisse aktueller Arbeiten deuten darauf hin, dass Barrieren vor allem struktureller Natur sind, also in fehlenden Kompetenzen und den Arbeitsbedingungen der Pflegenden liegen (Kajermo et al., 2012). Zu den wichtigsten Barrieren zählen demnach Mangel an Wissen und Kompetenz in Bezug auf EBP, unzureichende Unterstützung im klinischen Setting, fehlende Zeitressourcen, anderweitige Prioritätensetzung der Organisation, fehlende EBP-Mentor(inn)en sowie die mangelnde Autorität Pflegender, die Praxis ändern zu können. Ähnlich zeigen drei Erhebungen aus der deutschsprachigen Schweiz (Saxer, 2002; Schlaefli, 2005) sowie aus Deutschland (Schubert \& Wrobel, 2009), jeweils durchgeführt mit der BARRIERS Scale (Funk, Champagne, Wiese \& Tornquist, 1991), die Bedeutung struktureller Barrieren. In der Arbeit von Schubert und Wrobel (2009) erwiesen sich beispielsweise die Faktoren «Probleme mit dem Verständnis englischsprachiger Forschungsarbeiten», «fehlende positive Haltung der Krankenhäuser zu EBP» sowie «mangelnde Befugnisse Pflegender, Versorgungsroutinen zu ändern» als die am häufigsten genannten Barrieren.

Eine in Deutschland und Österreich grundsätzlich vorhandene Barriere ergibt sich aus der geringen Forschungsorientierung der größtenteils nichtakademischen Ausbildungen in der Pflege. Folgen der unzureichenden wissenschaftlichen Vorbildung sind vermutlich ein geringes Verständnis für die Notwendigkeit von Pflegeforschung und deren niedrigere Akzeptanz (Meyer \& Köpke, 2012).
Neben der Beschreibung von Barrieren gibt es international auch zu den fördernden Faktoren bei der Implementierung einer EBP weitreichende Forschungsaktivitäten. Hierzu wurde eine Reihe von Instrumenten entwickelt, um Bedingungsfaktoren für eine EBP und deren tatsächliche Umsetzung zu erfassen. Eine aktuelle systematische Übersichtsarbeit (Squires, Estabrooks, O'Rourke, Gustavsson, Newburn-Cook \& Wallin, 2011b) identifizierte für den Zeitraum von 1975 bis 2010 insgesamt 108 Publikationen zu 60 Instrumenten, die «research utilization» erfassen, davon allein 38 aus den letzten fünf Jahren. Eine Analyse von 55 Publikationen zur Erfassung der Implementierung von EBP, hauptsächlich aus den USA ( $\mathrm{n}=39$ ) und Europa $(\mathrm{n}=12)$, ergab, dass Pflegende berichten, in recht hohem Maße («moderate high») forschungsbasiert zu arbeiten (Squires, Hutchinson, Bostrom, O'Rourke, Cobban \& Estabrooks, 2011c). Durch eine weitere Analyse von 45 Publikationen (Squires, Estabrooks, Gustavsson \& Wallin, 2011a) konnte die Einstellung Pflegender zu Forschung als ein wichtiger individueller Faktor für die Nutzung von Forschung in der Praxis identifiziert werden. Außerdem wurden unter anderem die Teilnahme an Kongressen und internen Fortbildungen, eine akademische Ausbildung sowie das Ausmaß der Arbeitszufriedenheit als fördernde Faktoren dargestellt. Aus dem deutschsprachigen Raum waren für die genannten systematischen Übersichtsarbeiten keine Arbeiten verfügbar. Darüber hinaus ist die Übertragbarkeit der Ergebnisse zumindest auf Deutschland und Österreich zum Beispiel angesichts der bislang geringen akademischen Verortung der Pflege fraglich.

Zwei aktuelle Surveys aus Österreich (Breimaier et al., 2011) und Deutschland (Schnittger, Hilgefort \& Hauken, 2012) liefern erste Hinweise zum
Kenntnisstand der Pflegenden zu EBP sowie zum Interesse und zur Motivation, wissenschaftliche Ergebnisse in den Arbeitsalltag zu integrieren. Breimaier et al. (2011) befragten alle Pflegenden eines Universitätsklinikums in Österreich anhand des Instruments von Parahoo (1998). Von 1825 angeschriebenen Pflegenden antworteten 1023 (56\%). Nur 4,5\% der Befragten berichteten, häufig oder immer auf der Basis aktueller Forschung zu arbeiten, lediglich 12 Befragte $(1,2 \%)$ verfügten nach eigener Ansicht über ein ausreichendes Wissen, um forschungsbasiert arbeiten zu können. Die Einstellung zu EBP war tendenziell negativ. Nur ein Drittel der Befragten war der Meinung, dass Pflege ein auf Forschung basierender Beruf sein sollte. Vor kurzem publizierten Schnittger et al. (2012) die Ergebnisse einer Befragung von Pflegenden in drei niedersächsischen Krankenhäusern mittels eines selbst entwickelten Instruments. Von 1211 angeschriebenen Pflegenden antworteten 521 (43\%). Auch hier zeigt sich eine prinzipiell skeptische Haltung gegenüber EBP. So stimmten beispielsweise jeweils weniger als $50 \%$ der Befragten den Aussagen zu, dass «der Wissenschaftstransfer das Patient(inn)en-Outcome verbessert» und dass es in der eigenen Pflegepraxis «Handlungen oder Tätigkeiten» gibt, «die der wissenschaftlichen Forschung bedürfen».

Insgesamt zeigen die zitierten internationalen Arbeiten, dass sowohl individuelle als auch institutionelle Faktoren für die erfolgreiche und anhaltende Umsetzung von EBP von Bedeutung sind, wobei unklar bleibt, welche einen größeren Stellenwert haben. Die vorliegenden Ergebnisse aus Deutschland (Schubert \& Wrobel, 2009; Schnittger et al., 2012) geben Hinweise auf wichtige förderliche und hinderliche Faktoren für die Etablierung einer EBP. Sie legen nahe, dass Pflegende sich hierzu- 
lande ungenügend in der Lage und auch kaum dazu ermächtigt sehen, ihr Handeln nach den Prinzipien der EBP auszurichten und dass ein beträchtlicher Teil forschungsbasiertem Wissen keine wichtige Rolle für das eigene Handeln zuweist. Die Repräsentativität der Befunde ist jedoch limitiert, da sich die Arbeiten auf wenige, nicht zufällig ausgewählte Kliniken beschränken und die Rücklaufquoten nicht zufriedenstellend waren.

In Deutschland werden seit einiger Zeit vermehrt die Voraussetzungen und Bedingungen für eine EBP und die Rolle Pflegender diskutiert (Meyer \& Köpke, 2012). Beschriebene Unsicherheiten im Umgang mit englischsprachiger Literatur (Schubert \& Wrobel, 2009) sowie die mehrheitlich negativ gefärbte Haltung gegenüber forschungsbasiertem Wissen (Schnittger et al., 2012) können Indiz und Folge der hierzulande geringen akademischen Tradition des pflegerischen Berufs und damit einhergehender Rollenzuschreibungen sein. Kürzlich haben in Deutschland sowohl der Forschungsrat (2012) als auch der Wissenschaftsrat (2012) in Stellungnahmen die Notwendigkeit akademisch ausgebildeter Pflegender dargestellt. Für die Planung entsprechender Studienangebote fehlt es jedoch bislang an verlässlichen Informationen zur Studienbereitschaft und zu den Studieninteressen Pflegender. Darüber hinaus mangelt es an aussagekräftigen Erhebungen zu Einstellungen Pflegender in Deutschland, die vermutlich eine zentrale Komponente der erfolgreichen Implementierung einer EBP darstellen.

Ziel

Ziel dieser Studie war eine repräsentative Erhebung der Einstellung von Pflegenden zu EBP und ihrer Bewertung verschiedener Bedingungen für eine
EBP sowie von Aspekten der Akademisierung Pflegender als Grundlage einer EBP.

\section{Methode und Material}

\section{Forschungsdesign}

Es wurde eine querschnittliche multizentrische Befragung von Pflegenden in zufällig ausgewählten norddeutschen Krankenhäusern durchgeführt.

\section{Erstellung des Fragebogens}

International liegt eine Reihe von Instrumenten vor, die intendieren, Bedingungsfaktoren für eine EBP beziehungsweise für eine Forschungsbasierung der Praxis («research utilization») zu erfassen. Von den 60 Instrumenten in der systematischen Übersichtsarbeit von Squires et al. (2011b) waren sieben umfassend validiert und in mehr als einer Studie untersucht worden. Angesichts der Situation in Deutschland erschien keines der vorliegenden Instrumente vollständig übertragbar, so dass für diese Erhebung ein eigener Fragebogen entwickelt wurde, der einerseits Teile der bereits genannten Instrumente und andererseits eigene Items enthält. Auf der Grundlage eines Expert(inn)enkonsens (SK, FK, KB) wurden aus drei der am häufigsten benutzten Instrumente (Squires et al., 2011b) passende Items beziehungsweise Subskalen ausgewählt:

1) Edmonton Research Orientation Survey (EROS) (McCleary \& Brown, 2002; Pain, Hagler \& Warren, 1996)

2) Parahoo Instrument (Parahoo, 1998)

3) Estabrooks Instrument (Estabrooks, 1999; Estabrooks, Scott, Squires, Stevens, O'Brian-Pallas, Watt-Watson et al., 2008).

Die Auswahl erfolgt vor allem auf Grundlage der definierten Problemstellung, der Validität der Instrumente sowie der Anwendbarkeit auf die Situation in Deutschland. Die Autorinnen der Originalfragebögen wurden angeschrieben und um Zusendung der Instrumente gebeten. Das Parahoo-Instrument lag bereits in der autorisierten Übersetzung von Breimaier et al. (2011) vor und Teile hieraus wurden mit Genehmigung der Autorin (H. Breimaier) übernommen. Teile des EROS sowie des Estabrooks-Instruments wurden durch zwei Autor(inn)en (SK, FK) ins Deutsche und durch einen englischen Muttersprachler zurück ins Englische übersetzt. Die Ergebnisse wurden den Autorinnen der Instrumente mit Bitte um Bestätigung zugesandt und nach kleineren sprachlichen Modifikationen von diesen autorisiert.

Die Fragen des endgültigen Fragebogens lassen sich fünf übergeordneten Themen zuordnen:

1) Wissens- und Informationsgrundlagen

2) Bedeutung einer EBP

3) Voraussetzungen für eine EBP

4) Akademisierung in der Pflege

5) Demografische Angaben der Befragten.

\section{Pretestung des Fragebogens}

Eine erste vollständige Version des Fragebogens wurde zuerst vier Pflegebeziehungsweise Gesundheitswissenschaftler(inne)n mit der Bitte um kritische Rückmeldung vorgelegt. Hierbei stand im Besonderen die Augenscheinvalidität der eigens entwickelten Items im Fokus. Die Kommentare führten $\mathrm{zu}$ einer weiteren, hauptsächlich formalen Überarbeitung. Inhaltliche Änderungen waren lediglich in den selbst entwickelten Items nötig. In einem weiteren Schritt wurden vier Pflegende gebeten, im Rahmen von 30 - bis 60-minütigen kognitiven Interviews (Drennan, 2003; Faulbaum, Prüfer \& Rexroth, 2009) den Fragebogen zu lesen und die Fragen zu paraphrasieren. Außerdem wurde der Zeitaufwand erfragt. Die Interviews wurden aufgezeichnet und die Ergeb- 
nisse im Autor(inn)enteam diskutiert. Auf Grundlage der Interviews wurden weitere Modifikationen an den selbst entwickelten Items vorgenommen, die wiederum nicht den Inhalt der Originalinstrumente berührten.

Als letzter Schritt wurden der Fragebogen und das Vorgehen auf zwei Stationen getestet. Von 21 ausgegebenen Fragebögen waren 17 ausgefüllt zurückgesandt worden. Das Verfahren erwies sich als durchführbar und die Fragen als beantwortbar. Als Ergebnis wurden lediglich kleinere Modifikationen in der Ausfüllanweisung des Fragebogens vorgenommen. Die Ergebnisse der Pretest-Stationen wurden daher in die Endauswertung der Studie aufgenommen.

Der endgültige zehnseitige Fragebogen enthält insgesamt neun Fragen mit 63 Items sowie vier Items zur Demografie. Eine Fragebogenversion mit Hinweisen auf die zugrundeliegenden Instrumente ist auf Anfrage erhältlich.

\section{Rekrutierung der teilnehmenden Krankenhäuser und Stationen}

Geplant war eine Stichprobe von $n=20$ Krankenhäusern mit insgesamt 80 Stationen und etwa 1200 Teilnehmern in Hamburg und Schleswig-Holstein. Da die Studie explorativ angelegt war, gab es keinen validen Anhaltspunkt für eine Stichprobenkalkulation. Auch erlaubte die mangelnde Erfahrung mit dem Erhebungsinstrument keine Annahmen über die Werteverteilung in dieser Population. Daher wurde eine Stichprobe gewählt, die groß genug erschien, um hinreichend aussagekräftige Ergebnisse zu erreichen. Wichtige Aspekte hierbei waren die möglichst zufällige Auswahl der Teilnehmenden, eine heterogene Krankenhausauswahl sowie ein ausreichender Rücklauf.

Ziel war die Rekrutierung von dreizehn Häusern in Schleswig-Holstein und sieben Häusern in Hamburg. Um eine möglichst repräsentative Auswahl zu erreichen, wurden auf Basis vorliegender Klinikverzeichnisse Krankenhäuser zufällig nach Größe (Anzahl der Betten) und nach Bundesland stratifiziert ausgewählt. Dabei wurden für Schleswig-Holstein und Hamburg je vier Strata mit vorher festgelegten Krankenhausgrößen gebildet. Die Auswahl der Häuser erfolgte randomisiert mithilfe einer online erhältlichen Software (http://www.random.org/sequences). Da davon auszugehen war, dass nicht alle Häuser teilnehmen würden, wurden von vornherein mehr Häuser als die geplanten 20 Häuser ausgewählt und angeschrieben. Im März 2012 wurden insgesamt 22 Häuser (von 87) in Schleswig-Holstein und 15 (von 18) in Hamburg angeschrieben und in der Folgewoche telefonisch kontaktiert. Bei Bedarf wurde das Vorhaben in einem persönlichen Gespräch vor Ort vorgestellt. Um einen Selektionsbias zu vermeiden, wurden die teilnehmenden Häuser gebeten, möglichst zufällig Stationen für die Teilnahme an der Befragung auszuwählen. Je nach Einrichtungsgröße sollte eine bestimmte Anzahl an Stationen teilnehmen (Universitätskliniken/große Häuser etwa $\mathrm{n}=8$, mittlere Häuser etwa $\mathrm{n}=4$, kleine Häuser etwa $\mathrm{n}=2$ )

Es wurden verschiedene Bemühungen zur Erhöhung des Rücklaufs getroffen, wie sie in einschlägigen Publikationen aufgeführt (Edwards, Roberts, Clarke, Diguiseppi, Wentz, Kwan et al., 2009) wurden. Hierzu gehörten ein enger persönlicher Kontakt zu den teilnehmenden Einrichtungen, die Ankündigung einer strukturierten Rückmeldung der Ergebnisse für alle Einrichtungen sowie eine Verlosung von drei Geldpreisen $(200,100$ und $50 €)$ unter den teilnehmenden Stationen, unabhängig von der Rücklaufrate.

\section{Vorgehen bei der Befragung}

Nachdem die teilnehmenden Stationen benannt worden waren, nahm das
Forscher(innen)team Kontakt mit einem Ansprechpartner/einer Ansprechpartnerin der Station auf. Teilnehmende waren alle laut Dienstplan während eines vorher bestimmten Zeitraums von sieben Tagen im Dienst befindlichen examinierten Pflegenden sowie Auszubildenden. Die Befragungen erfolgten im März und April 2012. Die Fragebögen wurden zusammen mit einer Studieninformation in einem frankierten adressierten Umschlag durch die Kontaktperson des Krankenhauses beziehungsweise der Station an die Pflegenden verteilt. Diese wurden gebeten, den Umschlag per Post zurückzusenden. Auf sieben Stationen wurde auf Wunsch eine verschlossene Sammelbox aufgestellt, die nach Ablauf des Erhebungszeitraums durch ein Mitglied des Forscher(innen)teams abgeholt wurde. Die Befragten hatten dennoch auch hier die Möglichkeit, den Bogen postalisch zurückzusenden. Auf dem Umschlag befand sich eine sechsstellige Kodenummer, die eine Zuordnung zu dem Krankenhaus (Ziffern 1 und 2) und der Station (Ziffern 3 und 4) ermöglichte. Die Ziffern 5 und 6 dienten der temporären Identifikation der Befragten. Zu diesem Zwecke wurde in der Klinik beziehungsweise auf der Station eine Kodeliste geführt, auf der die Kodenummern den Befragten zugeordnet waren. Diese Zuordnung diente lediglich der Möglichkeit für das Studienteam, bei fehlendem Rücklauf einmalig über die Kontaktpersonen in den Kliniken nachzufragen. Da dem Studienteam die Kodeliste nicht bekannt war und wiederum der Kontaktperson vor Ort die ausgefüllten Fragebögen nicht zugänglich waren, erfolgte die Auswertung somit anonymisiert. Nach Eintreffen der Fragebögen in der Studienzentrale wurde der Umschlag vernichtet und lediglich der Eingang des Bogens dokumentiert. Die ersten vier Ziffern der Kodenummer wurden auf dem Bogen notiert, um eine stati- 
onsspezifische Auswertung zur strukturierten Rückmeldung zu ermöglichen.

\section{Auswertung}

Die Dateneingabe erfolgte durch ein Mitglied des Forscherteams in eine SPSS-Datenbank (Version 20) und wurde nach Abschluss der Erhebung von zwei weiteren Personen stichprobenartig anhand von zufällig ausgewählten 10 Prozent der Fragebögen kontrolliert. Da hierbei keine Fehler gefunden wurden, wurde auf eine vollständige Kontrolle verzichtet. Bei der Auswertung aufgefundene Inkongruenzen konnten durch Kontrolle der Fragebögen behoben werden. Die Auswertung erfolgte ausschließlich deskriptiv anhand von Mittelwerten und Standardabweichungen. Auf statistische Tests wurde angesichts des explorativen Charakters der Studie verzichtet, allerdings eine Post-hoc-Subgruppenanalyse zum Vergleich der Ergebnisse für ältere und jüngere Befragte in Bezug auf die Bereitschaft zu studieren durchgeführt. Hierfür wurden per Mediansplit zwei gleich große Gruppen gebildet.

\section{Ethik und Finanzierung}

Das Studienprotokoll wurde der Ethikkommission der Universität zu Lübeck vorgelegt und positiv begutachtet (Ref.-Nr. 12-022). Die Studie wurde zum Teil durch das Norddeutsche Zentrum zur Weiterentwicklung der Pflege (NDZ) finanziert.

\section{Ergebnisse}

\section{Krankenhäuser und Stationen}

Von den 37 angeschriebenen Krankenhäusern nahmen insgesamt 21 (16 in Schleswig-Holstein und 5 in Hamburg) mit 92 Stationen teil. Als Gründe für eine Nichtteilnahme wurden angegeben: Mangel an Ressourcen ( $n=7)$, Skepsis gegenüber der Studienfrage beziehungsweise des Studienziels $(n=4)$

Tabelle 1: Überblick teilnehmende Krankenhäuser ( $n=21$ ).

\begin{tabular}{l|c}
\hline Schleswig-Holstein/Hamburg & $16 / 5$ \\
\hline $\begin{array}{l}\text { Bettenzahl } \\
\text { (Mittelwert der Gesamtbetten je Krankenhaus) }\end{array}$ & $8921(425 \pm 357)$ \\
\hline $\begin{array}{l}\text { Anzahl Stationen } \\
\text { (Mittelwert der teilnehmenden Stationen je Krankenhaus) }\end{array}$ & $92(4,4 \pm 2,6)$ \\
\hline Intensivstationen & 22 \\
\hline Psychiatrie & 17 \\
\hline Chirurgie & 10 \\
\hline Innere & 43 \\
\hline andere* & 8 \\
\hline
\end{tabular}

Absolute Zahlen und (wenn angegeben) Mittelwerte ( \pm SD).

$* 13$ weitere Disziplinen mit jeweils weniger als 7 Stationen.

sowie Ablehnung durch den Betriebsrat $(\mathrm{n}=1)$. Vier Krankenhäuser gaben keine Gründe an. Die Tabelle 1 gibt einen Überblick über die Charakteristika der Krankenhäuser und der Stationen.

\section{Pflegende}

Von 1384 verteilten Fragebögen wurden 1041 zurückgesandt. Von diesen waren 18 leer, sodass 1023 Bögen in die Auswertung aufgenommen wurden. Das entspricht einer Rücklaufquote von $74 \%$. Fünf Teilnehmende hatten die Kodenummer unkenntlich gemacht, diese Bögen konnten somit keiner Station und keinem Krankenhaus zugeordnet werden, wurden aber in die Auswertung aufgenommen.

Die Teilnehmenden waren im Mittel 39,3 $( \pm 11,5)$ Jahre alt. 24 Teilnehmende waren Auszubildende. Die examinierten Pflegenden hatten im Mittel vor 16,4 $( \pm 11,3)$ Jahren ihr Examen abgeschlossen. Ein Drittel hatte mindestens eine Fachweiterbildung absolviert. Die Mehrzahl der Teilnehmenden $(n=648,64,7 \%)$ gab als höchsten Schulabschluss einen Realschulabschluss an, 120 (12\%) Fachhochschulreife sowie 203 (20,2\%) Abitur.

Wissens- und Informationsgrundlagen Tabelle 2 zeigt die Ergebnisse zur Frage, wie häufig bestimmte Informations- beziehungsweise Wissensquellen in der eigenen pflegerischen Arbeit von Bedeutung sind. Am häufigsten werden hier die eigene Erfahrung, individuelle Informationen zu Patient(inn)en und Kolleg(inn)engespräche genannt. Informationen aus der Ausbildung werden häufiger als Grundlage angegeben als Informationen aus Fortbildungen und Leitlinien beziehungsweise Standards. Primärliteratur aus pflegerischen oder gar medizinischen Zeitschriften wird selten genutzt.

Dennoch geben 461 Befragte $(45,7 \%)$ an, während der letzten zwei Jahre aktuelle Forschungsergebnisse genutzt zu haben. Mit «weiß nicht» antworten 252 Befragte (24,9\%).

\section{Bedeutung einer EBP}

Die Ergebnisse bezüglich der Bereitschaft, Forschungsergebnisse, die dem bisherigen Wissen widersprechen, in die eigene Praxis zu integrieren, (Tabelle 3, linke Spalte), deuten darauf hin, dass viele Pflegende einer EBP durchaus positiv gegenüberstehen. Es zeigt sich jedoch eine deutliche Diskrepanz zwischen der eingeschätzten Bereitschaft, neue Erkenntnisse zu nutzen, und deren tatsächlicher Implementierung. Auch wenn grundsätzlich die Bereitschaft besteht, auf Basis aktuel- 
Tabelle 2: Basis der pflegerischen Praxis.

\begin{tabular}{l|l}
\hline Erfahrung & $4,2 \pm 0,6$ \\
\hline individuelle Informationen zu jedem Patienten & $4,1 \pm 0,7$ \\
\hline Austausch unter Kollegen & $3,9 \pm 0,6$ \\
\hline Besprechungen mit Ärzten oder Therapeuten & $3,9 \pm 0,8$ \\
\hline Informationen aus der Pflegeausbildung & $3,8 \pm 0,8$ \\
\hline Leitlinien und Standards & $3,7 \pm 0,9$ \\
\hline interne Fortbildungen und Kongresse & $3,5 \pm 0,9$ \\
\hline Intuition & $3,5 \pm 0,8$ \\
\hline seit Jahren bewährte Praxis & $3,5 \pm 0,8$ \\
\hline neu angeordnete Therapien und Medikamente & $3,3 \pm 0,9$ \\
\hline Informationen aus Lehrbüchern & $3,2 \pm 0,8$ \\
\hline was schon immer gemacht wurde & $2,9 \pm 0,9$ \\
\hline Artikel aus Pflegefachzeitschriften & $2,5 \pm 1,0$ \\
\hline Medien & $2,5 \pm 0,9$ \\
\hline Artikel aus medizinischen Fachzeitschriften & $2,4 \pm 0,9$ \\
\hline
\end{tabular}

Mittelwerte $( \pm$ SD); 1 = nie bis $5=$ immer

ler Forschungsergebnisse zu arbeiten, wird dies offenbar nicht regelhaft umgesetzt (Tabelle 3, rechte Spalte).

Abbildung 1 zeigt, dass Forschung prinzipiell eine große Bedeutung beigemessen wird. So werden die beiden negativ gepolten Fragen zur Bedeutung von Forschung für die Praxis eindeutig abgelehnt. 12,4\% ( $\mathrm{n}=126)$ der Befragten stimmen der Aussage zu, dass Forschung lediglich für die Ausbildung, nicht aber für die Praxis relevant sei (trifft eher/definitiv zu) (MW 2,0 $( \pm 1,0)$ ). Die Aussage, dass Forschung im Berufsalltag nicht relevant sei, wird von $24,6 \%(n=250)$ der Befragten bejaht (MW 2,3 $( \pm 1,2)$ ). Es zeigt sich jedoch auch, dass die Befragten der Aussage eher nicht zustimmen, dass ein Großteil der Pflegenden über aktuelle Forschungsergebnisse informiert ist (Mittelwert 2,3 $( \pm 0,9))$ und die Umsetzung von Forschungsergebnissen in der Praxis als begrenzt angesehen wird
(Mittelwert 3,2 $( \pm 1,0))$. Die weiteren Fragen nach der Bedeutung von Forschung für die Praxis werden prinzipiell eher positiv beantwortet. So lehnen nur $22 \%(\mathrm{n}=223)$ die Aussage ab, dass Pflege ein auf Forschung beruhender Beruf werden solle (Mittelwert 3,7 $( \pm 0,9))$. Abbildung 1 zeigt darüber hinaus, dass Zeitmangel als wichtige Barriere bei der Information über Forschungsergebnisse und bei der Implementierung von Forschung angesehen wird.

Die Ergebnisse dreier Fragen zur Bedeutung von Forschung für die eigene Pflegepraxis fallen eher positiv aus. Auf einer fünfstufigen Likertskala von 1 (nein, gar nicht) bis 5 (ja, sehr) wird die Frage, ob pflegewissenschaftliche Studien Ergebnisse liefern, die «wirklich relevant» für die eigene Arbeit sind, deutlich positiv beantwortet (Mittelwert 3,8 $( \pm 1,0))$. Die Fragen nach der einfachen und sicheren Anwendbarkeit werden nur knapp positiv bewertet (Mittelwerte 3,2 $( \pm 1,0)$ beziehungsweise $3,2( \pm 0,9))$.

Auch die in Tabelle 4 dargestellten Ergebnisse zur Einstellung in Bezug auf die Einführung einer EBP zeigen prinzipiell positive Einstellungen.

\section{Voraussetzungen für eine EBP}

Bei den Fragen nach dem Stellenwert verschiedener Vorrausetzungen für eine EBP (Tabelle 5) zeigt sich ein ausge-

Tabelle 3: Bereitschaft zur Implementierung neuen Wissens.

\begin{tabular}{|l|l|l|}
\hline Zeitpunkt & $\begin{array}{l}\text { Bereitschaft, Forschungsergebnisse in die Praxis } \\
\text { einzuführen, die dem widersprechen, was zu } \\
\text { verschiedenen Zeitpunkten gelernt wurde. }\end{array}$ & $\begin{array}{l}\text { Häufigkeit, mit der auf Basis von Forschungs- } \\
\text { ergebnissen gearbeitet wird, die dem wider- } \\
\text { sprechen, was zu verschiedenen Zeitpunkten } \\
\text { gelernt wurde. }\end{array}$ \\
\hline vor der Ausbildung & $4,0 \pm 1,2$ & $2,9 \pm 1,3$ \\
\hline während der Ausbildung & $3,9 \pm 2,0$ & $2,8 \pm 1,1$ \\
\hline am Arbeitsplatz & $3,9 \pm 1,0$ & $2,7 \pm 1,1$ \\
\hline & $1=$ nein, gar nicht bis $5=$ ja, sehr & $1=$ nie bis $5=$ sehr oft \\
\hline
\end{tabular}


Tabelle 4: Einstellung zu EBP.

\begin{tabular}{|l|l|}
\hline Neue Ideen für die Praxis sind spannend. & $4,4 \pm 0,7$ \\
\hline $\begin{array}{l}\text { Auf dem neuesten Informationstand zu sein, ist ein wichtiger } \\
\text { Teil meiner Arbeit. }\end{array}$ & $4,1 \pm 0,9$ \\
\hline $\begin{array}{l}\text { Ich mag es, Tätigkeiten auf neue Art anzugehen und in meine } \\
\text { Arbeit einzubauen. }\end{array}$ & $4,0 \pm 0,8$ \\
\hline $\begin{array}{l}\text { Ich bin in der Lage, pflegerische Verfahren neu zu entwickeln } \\
\text { oder bestehende zu verändern, damit sie meinen Patienten } \\
\text { helfen. }\end{array}$ & $3,8 \pm 1,1$ \\
\hline $\begin{array}{l}\text { Ich suche ständig nach neuen Informationen, die für meine } \\
\text { Arbeit hilfreich sein könnten. }\end{array}$ & $3,6 \pm 1,2$ \\
\hline $\begin{array}{l}\text { Ich bin bereit, die Teilnahme an einer Tagung, die mich inter- } \\
\text { essiert, von meinem eigenen Geld zu bezahlen. }\end{array}$ & $2,7 \pm 1,3$ \\
\hline
\end{tabular}

Mittelwerte $( \pm$ SD); 1 = trifft definitiv nicht zu bis $5=$ trifft definitiv zu

prägter Deckeneffekt. Fast alle vorgeschlagenen Faktoren werden von den Befragten als wichtig eingeschätzt. Es fällt auf, dass Unterstützung (durch Vorgesetzte und andere) jeweils als vergleichsweise wichtig bewertet wird. Bei der Bedeutung eines pflegewissenschaftlichen Studiums zeigen sich die Befragten uneinig. Jeweils etwa ein Drittel hält ein pflegewissenschaftliches Studium für (eher) unwichtig oder (eher) wichtig oder ist unentschieden.

\section{Akademisierung in der Pflege}

Die Frage, ob sie sich vorstellen können, in den nächsten fünf Jahren ein pflegewissenschaftliches Studium zu beginnen, wird von der Hälfte der Teilnehmenden verneint (Abbildung 2). Lediglich jede/r fünfte Befragte hat dies fest vor. Als Gründe für die ablehnende Haltung gibt die Mehrzahl das eigene fortgeschrittene Alter und/ oder familiäre Verpflichtungen an. In einer Post-hoc-Subgruppenanalyse wurde daher das Antwortverhalten älterer und jüngere Befragter differenziert betrachtet. Die Gruppen unterscheiden sich deutlich. Während in der Gruppe derjenigen über 39 Jahre $60 \%$ $(\mathrm{n}=361$ ) für sich ein Studium in den nächsten 5 Jahren ausschließen, sind es bei den Unter-40-Jährigen nur $31 \%$ $(n=187)$. Hier finden sich darüber hinaus deutlich mehr Unentschiedene (Abbildung 2).

Auf die Frage nach Voraussetzungen zum Beginn eines Studiums werden finanzielle und organisatorische Aspekte als wichtig eingeschätzt. So ist die Bereitschaft gering, ein kostenpflichtiges Studium aufzunehmen ebenso wie ein Studium, das die zeitweilige Aufga- be der Berufstätigkeit erfordert (Mittelwerte [MW] 1,6 $( \pm 0,9)$ beziehungsweise $1,9( \pm 1,3)$ auf einer fünfstufigen Likertskala: 1 = keine Bereitschaft bis $5=$ hohe Bereitschaft, einen Studiengang aufzunehmen). Im Gegensatz dazu zeigt sich eine hohe Bereitschaft, einen vom Arbeitgeber/von der Arbeitgeberin finanzierten Studiengang $\mathrm{zu}$ beginnen (MW 4,5 $( \pm 1,1)$ ). Inhaltlich ist ein Studienangebot, das Pflegende für Schlüsselrollen in der täglichen Patientenversorgung qualifiziert, zum Beispiel als Expert(inn)en im Sinne von «Advanced Practice Nurses» für Intensivpflege, Onkologie oder Geriatrie, ebenfalls mit einer vergleichsweise hohen Bereitschaft verbunden (MW 3,8 $( \pm 1,3))$.

Mit einer einzelnen Frage wurde nach der prinzipiellen Einschätzung der Möglichkeit ausbildungsbegleitender Studiengänge in Deutschland gefragt. Nur $18,4 \%(\mathrm{n}=186)$ der Befragten stimmen der Aussage «Ich finde es wichtig, dass es jetzt auch in Deutschland möglich ist, gleichzeitig das Pflege-

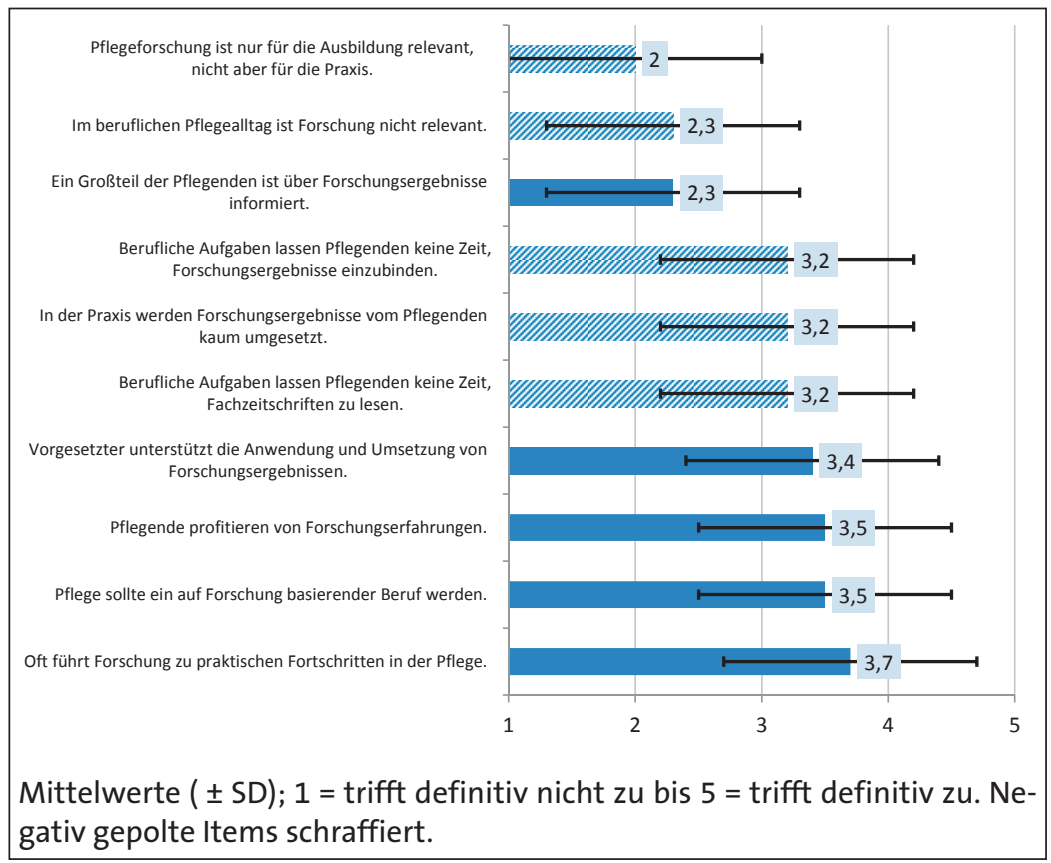

Abbildung 1: Bedeutung und Umsetzung von Forschung. 
Tabelle 5: Voraussetzungen für eine forschungsbasierte Praxis.

\begin{tabular}{l|l}
\hline Unterstützung durch Vorgesetzte & $4,7 \pm 0,6$ \\
\hline Pflegeexamen & $4,6 \pm 0,9$ \\
\hline Fort- oder Weiterbildungen zu Forschung & $4,5 \pm 0,8$ \\
\hline Unterstützung durch Kolleginnen und Kollegen & $4,4 \pm 0,8$ \\
\hline Unterstützung durch ärztliche Kolleginnen und Kollegen & $4,3 \pm 0,9$ \\
\hline Zugang zu Literaturdatenbanken am Arbeitsplatz & $4,2 \pm 1,0$ \\
\hline $\begin{array}{l}\text { Zugang zu Online-Plattformen mit aktuellen pflegewissenschaft- } \\
\text { lichen Erkenntnissen }\end{array}$ & $4,2 \pm 0,9$ \\
\hline $\begin{array}{l}\text { anteilige Freistellung für die Einführung neuer Erkenntnisse in } \\
\text { die Praxis }\end{array}$ & $4,1 \pm 0,9$ \\
\hline Angebot von EBN-Kursen & $4,0 \pm 1,0$ \\
\hline $\begin{array}{l}\text { anteilige Freistellung für die Suche nach aktuellen Forschungs- } \\
\text { ergebnissen }\end{array}$ & $3,9 \pm 1,1$ \\
\hline regelmäßige Treffen in Journal Club & $3,8 \pm 1,0$ \\
\hline langjährige Berufserfahrung & $3,5 \pm 1,2$ \\
\hline gute Englischkenntnisse & $3,4 \pm 1,2$ \\
\hline pflegewissenschaftliches Studium & $3,0 \pm 1,2$ \\
\hline
\end{tabular}

Mittelwerte $( \pm \mathrm{SD}) ; 1$ = unwichtig bis 5 = wichtig

examen und einen Bachelor-Abschluss in Pflege zu erwerben» (eher) nicht zu, $23,4 \%(n=237)$ sind unentschieden, $58,2 \%(\mathrm{n}=589)$ stimmen (eher) $\mathrm{zu}$.

\section{Diskussion}

Die vorliegende Studie berichtet die Ergebnisse einer Befragung von Pflegenden in Krankenhäusern in SchleswigHolstein und Hamburg zu Faktoren im Kontext der Implementierung einer EBP. Bisher lagen hierzu für den deutschsprachigen Bereich nur Arbeiten mit nicht-repräsentativen Kohorten vor (Breimaier et al., 2011; Schnittger et al., 2012). Im Gegensatz zu den Ergebnissen dieser Surveys zeigt die vorliegende Studie eine grundsätzlich positivere Einstellung gegenüber einer EBP. Die mit dem auch von Breimaier et al. (2011) verwendeten Parahoo-Instrument erhobenen Daten zur Bedeutung einer EBPI sind dabei mit internationa- len Befunden durchaus vergleichbar (Squires, Estabrooks, Gustavsson et al., 2011), stehen jedoch im Widerspruch zu den von Breimaier et al. (2011) erhobenen Befunden. So lehnen in der vorliegenden Befragung nur $22 \%$ die Aussage ab, dass Pflege ein auf Forschung beruhender Beruf werden solle. In der Erhebung von Breimaier et al. (2011) war dies knapp die Hälfte der Befragten $(49,2 \%)$. Große Unterschiede zeigen sich auch bezüglich der Grundlagen der Pflegepraxis. Während in der Erhebung von Breimaier et al. (2011) lediglich $13,2 \%$ der Befragten die Nutzung aktueller Forschungsergebnisse in den letzten zwei Jahren berichteten, waren es hier 45,7\%. In diesem Kontext kann auch die mehrheitlich geäußerte Bereitschaft, neues Wissen für die eigene Pflegepraxis zu nutzen (auch wenn dieses bisher Gelerntem widerspricht), als Offenheit für eine EBP gedeutet werden. Dieses Ergebnis macht sicher Mut für zukünftige Bestrebungen, Pfle- gende in Deutschland auf Basis der neuesten wissenschaftlichen Erkenntnisse aus- beziehungsweise weiterzubilden.

Auch wenn die Bedeutung von Forschung durch die befragten Pflegenden grundsätzlich als positiv für die eigene Praxis eingeschätzt wird, lassen die Ergebnisse jedoch an der aktuellen Umsetzung und der zukünftigen Umsetzbarkeit einer EBP in Deutschland zweifeln. Die in Tabelle 3 dargestellten Ergebnisse deuten auf eine Diskrepanz zwischen der Bereitschaft und der tatsächlichen Umsetzung einer EBP hin. Weitere Ergebnisse weisen ebenso in diese Richtung. So ist beispielsweise die Bereitschaft, eigene Zeit und Mittel in die Etablierung einer EBP zu investieren, wenig ausgeprägt. Nur eine Minderheit (35\%) der Befragten ist bereit, die Teilnahme an einer interessanten Tagung aus eigenen Mitteln zu finanzieren. Dies entspricht den Ergebnissen der Erhebung von Breimaier et al. (2011), bei der neben Zeitmangel und Mangel an Informationen beziehungsweise Wissen «Desinteresse» als eine der wichtigsten Barrieren für eine EBP genannt wurde. Bei den in Tabelle 4 dargestellten Ergebnissen zur Einstellung gegenüber EBP fällt auf, dass die Items, die konkret nach einer aktiven Verpflichtung fragen, wie der Verwendung eigener Geldmittel zur Finanzierung von Fortbildungsaktivitäten, negativer beantwortet werden als allgemeinere Aussagen zur Einführung von Neuerungen. Dennoch wird deutlich, dass Innovationen nicht abgelehnt, sondern prinzipiell positiv bewertet werden. Der Mittelwert der Mittelwerte der hier abgebildeten Subskala des EROS-Instruments von 3,77 ist vergleichbar mit Ergebnissen internationaler Erhebungen (Henderson, Winch, Holzhauser \& De Vries, 2006; McCleary \& Brown, 2002).

Ein weiterer interessanter Aspekt der vorliegenden Arbeit sind die Ergebnis- 


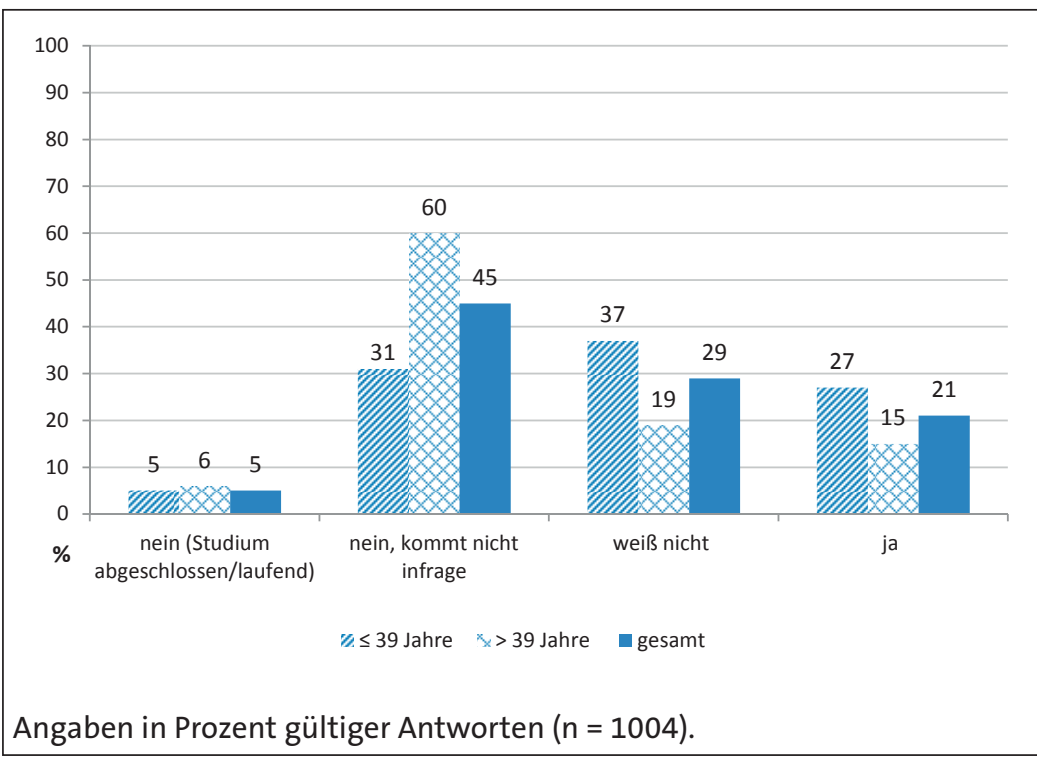

Abbildung 2: Eigenes pflegewissenschaftlichen Studium vorstellbar.

se $\mathrm{zu}$ den Voraussetzungen für eine EBP und hier die offensichtlich als nicht ausreichend empfundenen Kompetenzen und das unzureichende Wissen in Bezug auf eine EBP. So wird der Umgang mit Ergebnissen pflegewissenschaftlicher Studien nur von knapp der Hälfte der Befragten als anwendbar und sicher erachtet, obwohl die Mehrzahl diese als relevant für die eigene Praxis einschätzt. Die Informiertheit der Pflegenden wird als gering eingeschätzt. Es scheint also nicht an der Bereitschaft und Wertschätzung in Bezug auf eine EBP zu mangeln, jedoch bedarf es offensichtlich weiterer Kompetenzen, wie der Fähigkeit sich relevante Informationen $\mathrm{zu}$ erschließen und diese kritisch bewerten zu können, um auf Basis aktueller Forschung arbeiten zu können. Die berichteten Grundlagen der derzeitigen Praxis deuten an, dass dies zurzeit nicht der Fall ist. Auffällig ist, dass hier eher «klassische» Wissensquellen genannt werden. Natürlich müssen diese, also zum Beispiel die klinische Expertise, die Patient(inn)enbeobachtung und der kollegiale Austausch wichtige Grund- lagen der klinischen Praxis bleiben. Die Ergebnisse zeigen jedoch, dass der Umgang mit Primärquellen und mit schriftlichen Informationen im Allgemeinen einen niedrigen Stellenwert niger als der Hälfte der Befragten als häufige Grundlage der Praxis angesehen. Fachartikeln wird der gleiche Stellenwert zuerkannt wie Populärmedien (wie Zeitschriften, Fernsehen und Internet). Die Ergebnisse von Breimaier et al. (2011) bestätigen diesen Befund. Hier gaben $85 \%$ der Befragten an, höchsten alle zwei Monate Forschungsberichte oder -artikel zu lesen. Diese Ergebnisse stehen im deutlichen Gegensatz zu internationalen Befunden. In der systematischen Übersichtsarbeit von Spenceley, O'Leary, Chizawsky, Ross und Estabrooks (2008), in die 28 Arbeiten, hauptsächlich aus dem englischsprachigen Raum, eingeschlossen wurden, wird nach dem Austausch mit anderen Pflegenden das Wissen aus Pflegezeitschriften als zweitwichtigste Grundlage der Praxis dargestellt. Die Pflegeausbildung zeigte sich hier im Gegensatz zur vorliegenden Befragung hat. Selbst Lehrbücher werden von we- von untergeordneter Bedeutung. Angesichts einer mittleren Zeit seit Abschluss der Ausbildung von über 16 Jahren überrascht, dass die Mehrheit der Befragten Informationen aus der Ausbildung als derart wichtig ansieht. Ebenfalls denkwürdig ist die Bedeutung bestimmter Voraussetzungen für die erfolgreiche Etablierung einer EBP. Die Unterstützung durch Vorgesetzte und Kolleg(inn)en wird im Mittel als wichtiger empfunden als eine explizit für EBP-Arbeiten vorgehaltene anteilige Freistellung. Obwohl Zeitmangel durchaus als eine relevante Barriere wahrgenommen wird, scheinen Angebote, die speziell dieses Problem adressieren, eine etwas geringere Bedeutung zu haben als Strategien, die die EBPKultur fokussieren. Internationale Erfahrungen zeigen beispielsweise, dass sogenannte «Leadership»-Strategien (Gifford, Davies, Edwards, Griffin \& Lybanon, 2007) die Implementierung von EBP offenbar fördern können (RycroftMalone, 2008). Als Grundvoraussetzung einer forschungsbasierten Praxis wurde zum Beispiel das Vorhandensein von «leaders», also entsprechend ausgebildeten und motivierten Pflegenden, auch unter den «frontline nurses», also den klinisch Pflegenden beschrieben (Davies, Edwards, Ploeg, Virani, Skelly \& Dobbins, 2006). Das Ausbildungsniveau, die Möglichkeit zur Beteiligung an Forschung sowie die Einstellungen der Praktiker(innen) wurden ebenfalls als wichtigen Prädiktoren für das Ausmaß an EBP-Umsetzung identifiziert (Lizarondo, Grimmer-Somers \& $\mathrm{Ku}$ mar, 2011).

Die dargestellten Erkenntnisse erscheinen von besonderer Bedeutung bei der Etablierung einer EBP in Deutschland und deren Einbezug in Curricula bestehender und geplanter (Weiter-)Bildungsangebote für Pflegende. Die Ergebnisse deuten darauf hin, dass es neben der (hier nicht in den Fokus genommenen) Beseitigung von Barrieren 
sowie der Etablierung einer EBPfreundlichen «Praxiskultur» der deutlicheren Ausrichtung der Aus- und Weiterbildung auf die Förderung einer EBP bedarf. Bisherige Versuche, dies im Rahmen von bestehenden Ausbildungscurricula zu realisieren, scheinen wenig erfolgreich zu sein, wie die Erhebungen von Schnittger et al. (2012) und Breimaier et al. (2011) zeigen. Eine (grundständige) akademische Ausbildung könnte hier die nötigen Rahmenbedingungen schaffen. Die Studie liefert wichtige Aspekte für die Ausgestaltung dieser Studiengänge sowie für weitere Forschung. Pflegepädagogische Studiengänge sollten demnach ein besonderes Gewicht auf grundlegende EBP-Kompetenzen und deren Vermittlung legen, während Pflegemanager(inne)n die herausragende Bedeutung von Leadership-Kompetenzen und deren Ausgestaltung zu vermitteln ist. Für einen eigenen geplanten grundständigen Studiengang klinischer Pflegeexpert(inn)en ergeben sich weitere Aspekte. Als wichtige potenzielle Barriere bei der Etablierung einer akademischen Ausbildung in der Pflege wurde die eingeschränkte Akzeptanz akademisch ausgebildeter Pflegender in der Praxis beschrieben (Bormann, 2011). Die in dieser Studie generierten Befunde zeichnen ein positiveres Bild. So wurde die Frage nach der Bedeutung grundständiger Bachelorstudiengänge von einer deutlichen Mehrheit der Befragten bejaht. Allerdings waren sich die Befragten uneinig bezüglich der Bedeutung eines Studiums für eine EBP. Ein eigenes Studium wollten nur etwa $20 \%$ der Befragten in Zukunft sicher aufnehmen, bei einer großen Zahl Unentschlossener. Ein Grund für diese zurückhaltende Einschätzung mag, neben dem Alter, der derzeit noch nicht eindeutigen klinischen Ausrichtung solcher Studiengänge und der Abwesenheit akademisch ausgebildeter Pflegender in der klinischen Praxis ge- schuldet sein. Die vorliegende Studie liefert zumindest Hinweise darauf, dass akademische Ausbildungen, die über die bislang vorherrschenden eher praxisfernen Studiengänge hinausgehen, wertgeschätzt werden. Es zeigte sich eine hohe Bereitschaft, ein Studium zu beginnen, welches für Schlüsselrollen in der klinischen Versorgung qualifiziert. Diese Studie hat einige wichtige Stärken. Das gewählte Vorgehen zielte auf eine möglichst hohe Rücklaufrate und die Gewinnung einer repräsentativen Gruppe Pflegender. Beide Ziele konnten erreicht werden. Eine Rücklaufrate von $74 \%$ ist bislang in keiner ähnlich großen Studie zu den Rahmenbedingungen einer EBP-Implementierung erreicht worden. Auch wenn es keinen etablierten Grenzwert für Rücklaufquoten gibt, so werden gemeinhin doch mindestens $60 \%$ Rücklauf als untere Grenze angesehen (Johnson \& Wislar, 2012). Neben der hohen Rücklaufquote lassen sowohl das gewählte Vorgehen der stratifizierten Rekrutierung der Krankenhäuser als auch der Auswah der teilnehmenden Stationen und Pflegenden auf repräsentative Ergebnisse zumindest für Schleswig-Holstein und Hamburg schließen.

Die Arbeit hat allerdings auch einige Limitierungen. Angesichts der zahlreichen vorliegenden etablierten Instrumente wurde auf die Entwicklung und Validierung eines eigenen Instrumentes verzichtet. Da keines der vorliegenden Instrumente in Gänze für die Situation in Deutschland angemessen schien, wurden Teile vorliegender etablierter Instrumente verwandt. Zur Akademisierung sowie zu Voraussetzungen für eine EBP wurden aufgrund der spezifischen Situation in Deutschland eigene Items entwickelt. Bei letzteren zeigte sich ein deutlicher Deckeneffekt. Angesichts des explorativen Charakters der Studie erfolgte die Darstellung der Ergebnisse lediglich deskriptiv. Es können somit keine Aus- sagen getroffen werden über einen möglichen Zusammenhang zwischen Charakteristika der Pflegenden, wie Ausbildungsstand und Alter, und deren Einstellung zu EBP und der Bereitschaft, EBP einzuführen. Es bleibt unklar, ob eine positive Haltung und/oder Kompetenzen tatsächlich eine EBP begünstigen. Daten aus internationalen Erhebungen deuten jedoch darauf hin, dass sowohl die Einstellung der Pflegenden (Wallin et al., 2012) als auch deren Ausbildungsgrad (Lizarondo et al., 2011) positiv mit einer EBP assoziiert sind. Die Repräsentativität der Studie ist eventuell eingeschränkt, da die Auswahl der Stationen den teilnehmenden Häusern überlassen wurde. Es wurde nicht geprüft, ob die Verteilung der teilnehmenden Stationen denen der teilnehmenden Häuser entspricht und ob die Charakteristika der teilnehmenden Häuser denen der Gesamtheit der Häuser in Schleswig-Holstein und Hamburg ähneln.

Zusammenfassend zeigt die Arbeit eine prinzipiell positive Einstellung Pflegender gegenüber einer EBP. Erwartungsgemäß zeigt sich jedoch eine deutliche Kluft zwischen Anspruch und Wirklichkeit. Es bedarf somit weiterer Anstrengungen, um die Prinzipien der EBP in der Pflegepraxis zu verankern, das heißt die oft anzutreffende «Aktionismuskultur» in Richtung einer «Fragekultur» zu verändern. Als wichtige und erfolgversprechende Maßnahmen erscheinen hierfür zum einen die Unterstützung der Pflegenden durch EBPaffine Führungspersonen ( «Leadership») sowie zum anderen die Anpassung der Aus- und Weiterbildung im Sinne einer EBP, inklusive des Angebotes klinisch orientierter Pflegestudiengänge.

\section{Dank}

Wir danken allen beteiligten Pflegenden und Krankenhäusern sowie 
Katharina Brinkmeier, Anne Junghans und Julia Wollmer für die Unterstützung bei der Datenerhebung und -eingabe. Außerdem Susanne Buhse, Anja Gerlach, Gabriele Meyer und Peter Nydahl für die kritische Revision des Fragebogens sowie Henry Holland für die Rückübersetzungen der englischsprachigen Items.

\section{German hospital nurses' attitudes concerning evidence-based nursing practice}

The relevance of nurses' attitudes for establishing an evidence-based nursing practice (EBP) has been proven internationally. For German-speaking countries so far only few data are available. The present survey aims at assessing nurses' perceptions of relevant context factors for implementing an EBP. Therefore, 1384 nurses in 21 hospitals in Northern-Germany received a self-developed questionnaire based on established instruments in March and April 2012. 1023 (74\%) nurses responded. In principal, results show a positive attitude towards EBP. The majority of participants regards research as relevant for nursing practice. Support from superiors and colleagues is seen as important prerequisite. However, implementation remains a challenge. Nurses are not informed about recent research results. Original articles are hardly used. Only a minority is prepared to spend own money on congresses or to start academic nursing training in the near future. For the first time in Germanspeaking countries, the study provides meaningful data on nurses' attitudes towards EBP. Nurses confirm the value of research for their own practice. However, there is a lack of basic requirements to identify and implement relevant research findings as for example the use of recent scientific evidence. Nursing education in Germany should therefore focus more strongly on building competencies required for $E B P$, for example through properly designed academic nursing training.

Key words: evidence-based practice, evidence-based nursing, research utilization, implementation, survey

\section{Literatur}

Behrens, J.; Langer, G. (Hrsg.) (2004) Evidence-based Nursing. Vertrauensbildende Entzauberung der Wissenschaft. Bern: Hans Huber.

Bormann, M. (2011). Aktueller Stand der Integration von Bachelor of Nursing Absolventinnen in die klinische Pflegepraxis. HeilberufeSCIENCE, 2 (Supplement 2), 40.

Breimaier, H.; Halfens, R.; Lohrmann, C. (2011). Nurses' wishes, knowledge, attitudes and perceived barriers on implementing research findings into practice among graduate nurses in Austria. J Clin Nurs, 20 (11-12), 1744- 1756.

Davies, B.; Edwards, N.; Ploeg, J.; Virani, T.; Skelly, J.; Dobbins, M. (2006). Determinants of sustained use of research evidence in nursing. www. chrsf.ca/Migrated/PDF/ResearchReports/OGC/davies_ final_e.pdf_[14.09.2012].

Drennan, J. (2003). Cognitive interviewing: verbal data in the design and pretesting of questionnaires. J Adv Nurs, 42 (1), 57 - 63.

Edwards, P.; Roberts, I.; Clarke, M.; Diguiseppi, C.; Wentz, R.; Kwan, I.; Cooper, R.; Felix, R.; Pratap, S. (2009). Methods to increase re- sponse to postal and electronic questionnaires. Cochrane Database Syst Rev (3).

Estabrooks, C. (1999). The conceptual structure of research utilization. Res Nurs Health, 22 (3), 203 - 216.

Estabrooks, C.; Scott, S.; Squires, J.; Stevens, B.; O'Brien-Pallas, L.; Watt-Watson, J.; Profetto-McGrath J.; McGilton K.; Golden-Biddle K.; Lander J.; Donner G.; Boschma G.; Humphrey C.; Williams, J. (2008). Patterns of research utilization on patient care units. Implement Sci, 3,31 .

Faulbaum, F.; Prüfer, P.; Rexroth, M. (2009). Was ist eine gute Frage? Die systematische Evaluation der Fragenqualität. Wiesbaden: VS.

Forschungsrat (2012). Forschung in den Gesundheitsfachberufen: Potenziale für eine bedarfsgerechte Gesundheitsversorgung in Deutschland. DMW, 137 (S2), S29-S76.

Funk, S; Champagne, M.; Wiese, R.; Tornquist, E. (1991). BARRIERS: the barriers to research utilization scale. Appl Nurs Res, 4 (1), 39-45.

Gifford, W.; Davies, B.; Edwards, N.; Griffin, P.; Lybanon, V. (2007). Managerial leadership for nurses' use of research evidence: an integrative review of the literature. Worldviews Evid Based Nurs, 4 (3), 126 - 145.

Henderson, A.; Winch, S.; Holzhauser, K.; De Vries, S. (2006). The motivation of health professionals to explore research evidence in their practice: An intervention study. J Clin Nurs, 15 (12), 1559 - 1564.

Hutchinson, A.; Johnston, L. (2004). Bridging the divide: a survey of nurses' opinions regarding barriers to, and facilitators of, research utilization in the practice setting. J Clin Nurs, 13 (3), 304-315.

Johnson, T.; Wislar, J. (2012). Response rates and nonresponse errors in surveys. JAMA, 307 (17), 1805 - 1806. 
Kajermo, K.; Bostrom, A.; Thompson, D.; Hutchinson, A.; Estabrooks, C.; Wallin, L. (2010). The BARRIERS scale - the barriers to research utilization scale: A systematic review. Implement Sci, 5, 32.

Köpke, S.; Mühlhauser, I.; Gerlach, A.; Haut, A.; Haastert, B.; Möhler, R.; Meyer, G. (2012). Effect of a guideline-based multicomponent intervention on use of physical restraints in nursing homes: a randomized controlled trial. JAMA, 307 (20), $2177-2184$.

Lizarondo, L.; Grimmer-Somers, K.; Kumar, S. (2011). A systematic review of the individual determinants of research evidence use in allied health. J Multidiscip Healthc, 4, 261 - 272.

McCleary, L.; Brown, G.; (2002). Use of the Edmonton Research Orientation Scale with nurses. J Nurs Meas, 10 (3), $263-275$.

Meyer, G.; Balzer, K.; Köpke, S. (2013). Evidenzbasierte Pflegepraxis - Diskussionsbeitrag zum Status Quo. Z Evid Fortbild Qual Gesundhwes, 107(1), 30-35.

Meyer, G., Köpke, S. (2012). Wie kann der beste pflegewissenschaftliche Kenntnisstand in die Pflegepraxis gelangen? Pflege und Gesellschaft, 17 (1), 36- 44.

Middleton, S.; McElduff, P.; Ward, J.; Grimshaw, J.; Dale, S.; D'Este, C.; Drury, P.; Griffiths, R.; Cheung, N.; Quinn, C.; Evans, M.; Cadilhac, D.; Levi, C.; QASC Trialists Group. (2011). Implementation of evidence-based treatment protocols to manage fever, hyperglycaemia, and swallowing dysfunction in acute stroke (QASC): a cluster randomised controlled trial. Lancet, 378 (9804), 1699 - 1706.
Pain, K.; Hagler, P.; Warren, S. (1996). Development of an instrument to evaluate research orientation of clinical professionals. Can. J. Rehabil, 9 (2), 93-100.

Parahoo, K. (1998). Research utilization and research related activities of nurses in Northern Ireland. Int J Nurs Stud, 35 (5), 283 - 291.

Retsas, A. (2000). Barriers to using research evidence in nursing practice. J Adv Nurs, 31 (3), 599-606.

Rycroft-Malone, J. (2008). Leadership and the use of evidence in practice. Worldviews Evid Based Nurs, 5 (1), $1-2$.

Saxer, S. (2002). Transfer von Forschungsergebnissen in die Praxis Hemmende und fördernde Faktoren. PrInterNet, (4), 17-23.

Schlaefli, Y. W. (2005). Einflussfaktoren bei der Anwendung von pflegewissenschaftlichen Erkenntnissen: Eine Untersuchung bei HochschulabsoventInnen in Pflegeberufen in der deutschsprachigen Schweiz. http://books.google.de/books?id= 7dvkSAAACAAJ

Schlömer, G. (2000). Evidence-based nursing. Eine Methode für die Pflege? Pflege, 13 (1), 47 - 52.

Schnittger, T.; Hilgefort, M.; Hauken, T. (2012). Status quo des Evidencebased Nursing. Pflegewissenschaft, (3), $140-150$.

Schubert, B.;Wrobel, M. (2009). Identifizierung von Hindernissen, die die Implementierung von Forschungswissen in die Pflegepraxis hemmen. Pflegewissenschaft, (6), $343-351$.

Sitzia, J. (2002). Barriers to research utilisation: the clinical setting and nurses themselves. Intensive Crit Care Nurs, 18 (4), 230 - 243.
Spenceley, S.; O'Leary, K.; Chizawsky, L.; Ross, A.; Estabrooks, C. (2008). Sources of information used by nurses to inform practice: An integrative review. Int J Nurs Stud, 45 (6), 954-970.

Squires, J.; Estabrooks, C.; Gustavsson, P.; Wallin, L. (2011a). Individual determinants of research utilization by nurses: a systematic review update. Implement Sci, 6, 1.

Squires, J.; Estabrooks, C.; O'Rourke, H.; Gustavsson, P.; Newburn-Cook, C.; Wallin, L. (2011b). A systematic review of the psychometric properties of self-report research utilization measures used in healthcare. Implement Sci, 6, 83.

Squires, J.; Hutchinson, A.; Bostrom, A.; O'Rourke, H.; Cobban, S.; Estabrooks, C. (2011c). To what extent do nurses use research in clinical practice? A systematic review. Implement Sci, 6, 21.

van Gaal, B.; Schoonhoven, L.; Mintjes, J.; Borm, G.; Koopmans, R.; van Achterberg, T. (2011). The SAFE or SORRY? programme. part II: effect on preventive care. Int J Nurs Stud, 48 (9), 1049- 1057.

Wallin, L.; Bostrom, A; Gustavsson, J. (2012). Capability Beliefs Regarding Evidence-Based Practice are Associated with Application of EBP and Research Use: Validation of a New Measure. Worldviews Evid Based Nurs, 9 (3), 139 - 148.

Wissenschaftsrat (2012). Empfehlungen zu hochschulischen Qualifikationen für das Gesundheitswesen. http://www.wissenschaftsrat.de/ download/archiv/2411-12.pdf [14.09.2012]. 
Was war die größte Herausforderung bei Ihrer Studie?

Die Erlangung eines angemessenen Rücklaufs.

Was wünschen Sie sich bezüglich der Thematik für die Zukunft?

Dass sich durch verbesserte Rahmenbedingungen die Einstellungen der Pflegenden in den nächsten Jahren deutlich ändern.

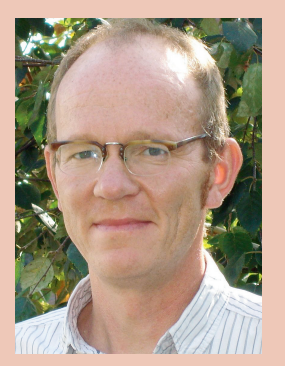

Was empfehlen Sie den LeserInnen zum Weiterlesen/Vertiefen?

Die Literatur hierzu ist immens. Besonders die kanadische Arbeitsgruppe um Estabrooks publiziert viel Interessantes. Von dort sind auch in Zukunft vielversprechende neue Ideen und Erkenntnisse zu erwarten.

\section{Korrespondenzadresse}

Prof. Dr. phil. Sascha Köpke Sektion Forschung und Lehre in der Pflege Institut für Sozialmedizin und Epidemiologie

Universität zu Lübeck

sascha.koepke@uksh.de 